Report No. Y/LB-16,160

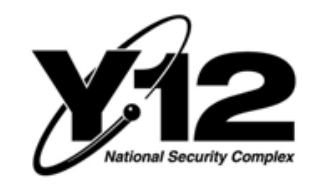

A BWXT/Bechtel Enterprise

\section{Addition of Tomographic Capabilities To NMIS}

J. A. Mullens

Y-12

National

Security

Complex Readiness in Technical Base and Facilities

MANAGED BY BWXT Y-12, L.L.C. FOR THE UNITED STATES DEPARTMENT OF ENERGY
Material Stewardship Program

Prepared by the Y-12 National Security Complex Oak Ridge, Tennessee 37831 managed by BWXT Y-12, L.L.C. for the

March 11, 2003 U.S. DEPARTMENT OF ENERGY
under contract DE-AC05-00OR22800 


\section{DISCLAIMER}

This report was prepared as an account of work sponsored by an agency of the United States government. Neither the United States Government nor any agency thereof, nor any of their employees, makes any warranty, express or implied, or assumes any legal liability or responsibility for the accuracy, completeness, or usefulness of any information, apparatus, product, or process disclosed, or represents that its use would not infringe privately owned rights. Reference herein to any specific commercial product, process, or service by trade name, trademark, manufacturer, or otherwise, does not necessarily constitute or imply its endorsement, recommendation, or favoring by the United States Government or any agency thereof. The views and opinions of authors expressed herein do not necessarily state or reflect those of the United States Government or any agency thereof. 


\section{Addition of Tomographic Capabilities to NMIS}

This paper describes tomographic capabilities for the Nuclear Materials Identification System (NMIS). The tomographic capabilities add weapons component spatial and material properties information that result in a more detailed item signature (template) and provide more information for physical attributes analyses.

\section{Introduction}

The Nuclear Materials Identification System (NMIS) is used routinely to confirm the identity of HEU components in sealed containers. It does this through a radiation signature acquired by shining a ${ }^{252} \mathrm{C}$ f source through the container and measuring the radiation at four detectors stacked vertically on the other side. This measurement gives a gamma and neutron radiation transmission profile of the weapons component, mixed with the radiation production due to the induced fissions in the fissile materials. This information is sufficient to match an 'unknown' weapons component signature to a template signature from a reference item when measuring under controlled conditions.

Tomography measures the interior of an item by making transmission measurements from all angles around the item, whereas NMIS makes the measurements from a single angle. Figure 1 is a standard example of tomographic reconstruction, the Shepp-Logan human brain phantom. The measured quantity is attenuation so high values (white) are highly attenuating areas.

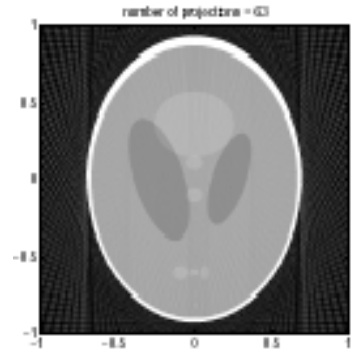

Figure 1 A common tomography example: scan of a simulated human brain (Shepp-Logan phantom).

The advantage to NMIS is more geometric and materials property information. The tomography capability provides more information about the geometry of the weapons component, which should lead to better interpretation of the radiation signature. The NMIS signature also has some ability to separate gamma rays and neutrons by time of flight (detector arrival time). This allows the system to construct a series of tomographic views of the weapon component's approximate gamma and neutron interaction properties. The combination of geometric and materials property information will result in better template matching and attributes analysis. It can also give images, and these are more easily interpreted by the NMIS operator than the current NMIS signatures.

The advantages that NMIS brings to tomography are its timed correlation/coincidence signature and its neutron/gamma point source. 


\section{Technical Basis}

\section{Example of a Tomographic Reconstruction Using the NMIS Signature}

This section presents MCNP-PoliMi ${ }^{1}$ simulations of a tomographic NMIS measurement. A hollow cylindrical HEU casting with a poly rod in the center was scanned to demonstrate the basic concept (see Figure 2). This item is difficult because the 0.8 " diameter poly rod is entirely within the 0.75 " thick walls of the HEU casting, and the wall of the steel can containing the casting is only 0.01 " thick. MCNP was used so that all of the complications of scattering and induced fission effects would be taken into account. A standard fan beam tomography configuration was used, with 391 " wide detectors in order to produce an "operator-usable" image with a resolution of about 0.2 ". . Since the ${ }^{252} \mathrm{Cf}$ is a point source it is close to the container and the detector array is placed far enough away to achieve the resolution desired. The fan angle is $112^{\circ}$, which is large enough to cause some problems in the imaging. There were no collimators or shields between detectors. These detectors could be, for example, a liquid scintillator tank with baffles separating the detector sections.

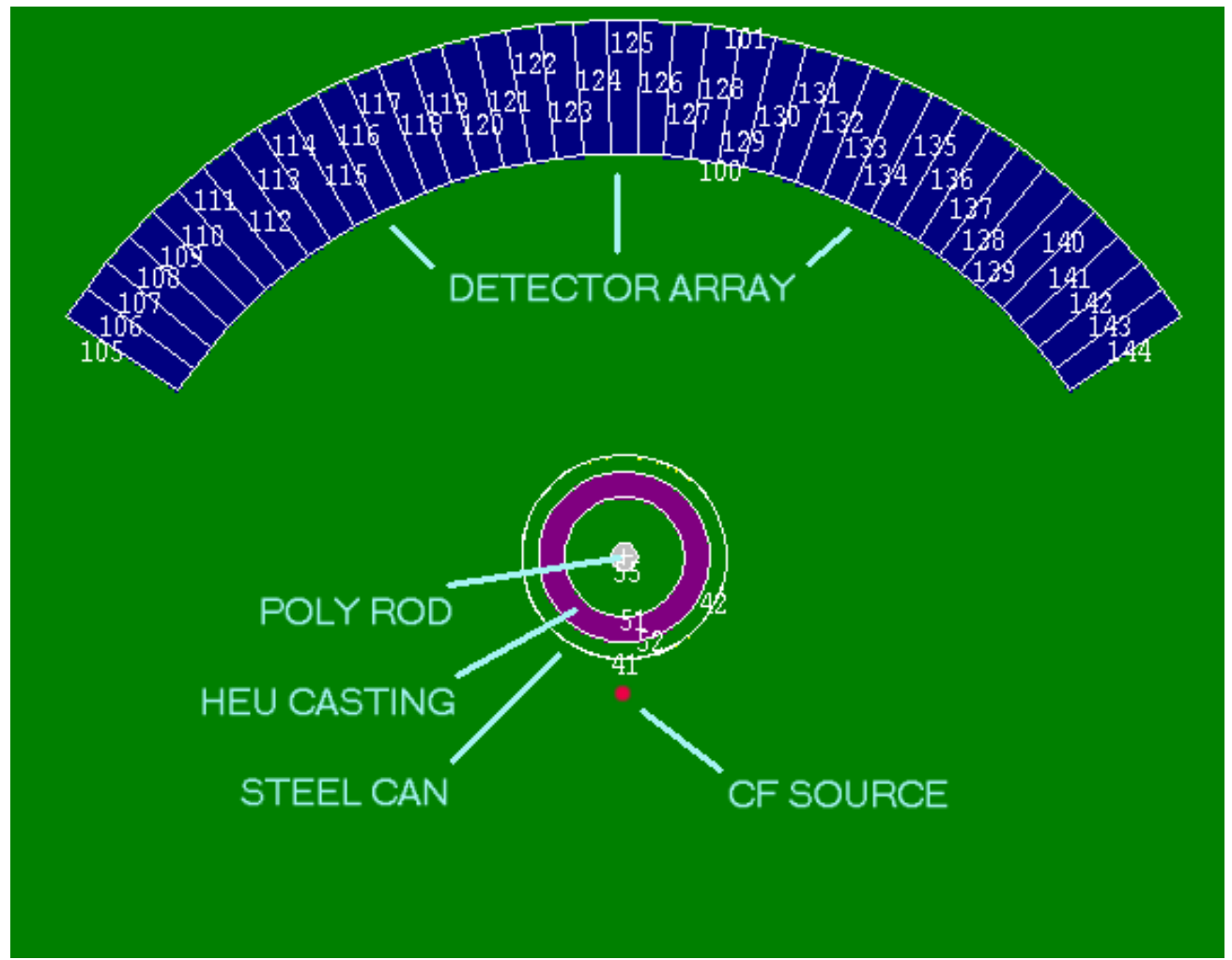

Figure 2 Overhead view of the beam arrangement of the simulated measurement.

\footnotetext{
${ }^{1}$ E. Padovani and S.A. Pozzi, "MCNP-PoliMi ver. 1.0. User's Manual", CESNEF-021125 Library of Nucl. Eng. Dept., Polytechnic of Milan, Italy, Nov. 2002.

${ }^{2}$ Either 39 actual detectors could be used or a small number of detectors could be rotated through these positions.
} 
The MCNP simulation ran for $50 \times 10^{6} \mathrm{Cf} \mathrm{fissions}^{3}$ to produce a projection measurement. This is a measurement through the item at one angle (rotation of the casting). Because MCNP simulations take a long time, a symmetric collection of objects was used and this projection was used for all angles. The measurements are very well converged at $50 \times 10^{6}$ Cf fissions, so the reconstructions shown here should give a good idea of the physical principles behind the measurement. Shorter, less converged measurements could be simulated using a converged measurement as the basis, by shortening it (fewer counts according to measurement time) and randomizing the number of counts (with standard deviation equal to the number of counts). However the point of this study is not to look at measurement time effects but the physics of the measurement.

The resulting NMIS correlation signature was divided into two regions: the directlytransmitted gamma rays which arrived first $(1-3 \mathrm{~ns})$, then all radiation which arrived between 15 and $25 \mathrm{~ns}$. The latter radiation is predominately source neutrons with a range of energies, so for the sake of brevity they will be referred to as neutrons. Other radiation includes induced fission neutrons and gammas, gammas from inelastic neutron scatter in the casting. A typical NMIS ${ }^{252} \mathrm{Cf}$ signature is shown in Figure 3 for the detector in the center of the detector array.

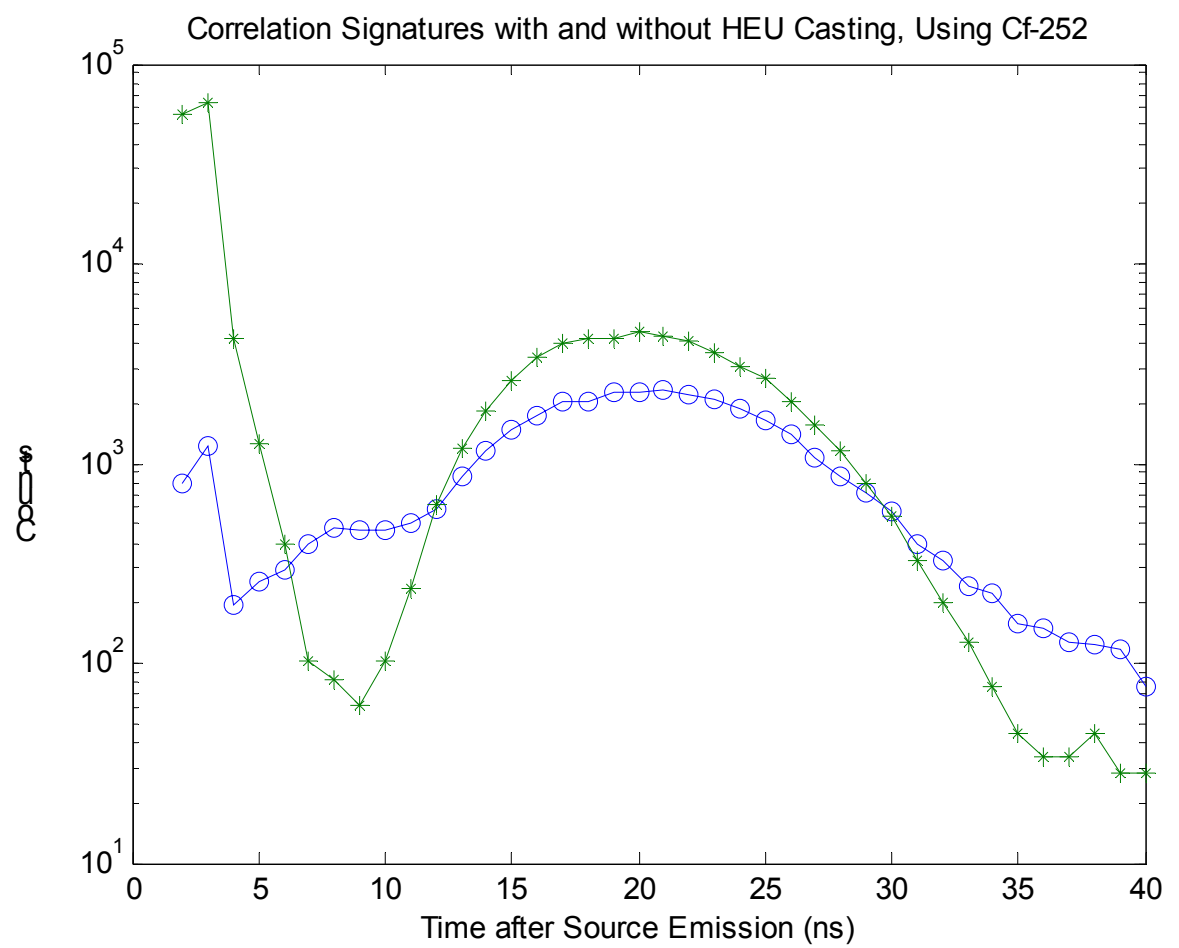

Figure 3 NMIS signature measured through the center of the scan area, intersecting all objects in the scan. Also shown is the signature with no objects in the scan, used to normalize the scan signature, which shows the detetor's response to the bare source.

${ }^{3}{ }^{252} \mathrm{Cf}$ sources are used with NMIS that produce in excess of $10^{6}$ fissions per second, so this projection would require less than one minute to measure in the field. Total measurement time would depend on the attenuation of the scanned item, number of projections required to achieve the desired resolution, and other factors. 
A typical NMIS DT generator signature is shown in Figure 4 for the detector in the center of the detector array. The void measurement in the figure shows the directly-transmitted neutrons arriving between 7 and 8 nanoseconds, and subsequent scattering between detectors. The casting measurement shows the directly-transmitted neutrons preceded by gamma rays generated by the initial contact of the source neutrons with the casting, and followed by radiation from induced fissions, scattering, etc.

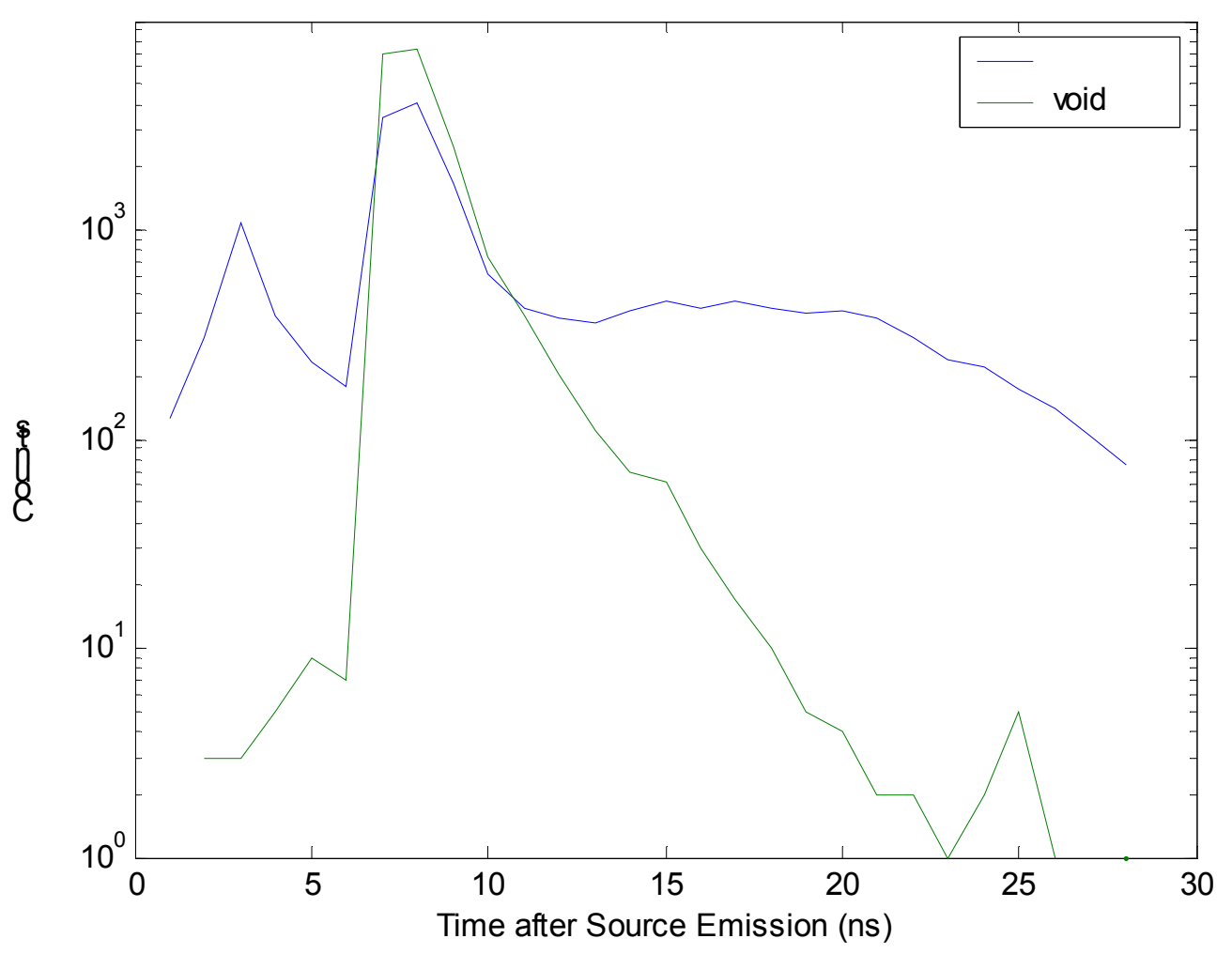

Figure 4 NMIS DT generator signature measured through the center of the scan area. Also shown is the signature with no objects in the scan, used to normalize the scan signature, which shows the detector's response to the bare source.

The detectors' response is shown in Figure 5 for the neutrons. The response, $\mathrm{S}$, is

$S=-\log \left(\frac{C}{C_{0}}\right)$

Equation 1

where $\mathrm{C}$ and $\mathrm{C}_{0}$ are the detector counts with and without the scanned object in place. Therefore $\mathrm{S}$ is proportional to the materials' cross sections, and higher values show more attenuation.

$\mathrm{S}$ has the characteristics expected, except that neutron scattering and other effects resulted in a $20 \%$ increase in counts into outlying detectors with the casting in place $\left(\mathrm{C} / \mathrm{C}_{0}\right)$. (The ray between the source and the end detectors does not pass through any of the objects.) 
The detector response in Figure 5 shows that the maximum attenuation occurs on the long path through the HEU side, rather than the path through the casting center (see Figure 6).

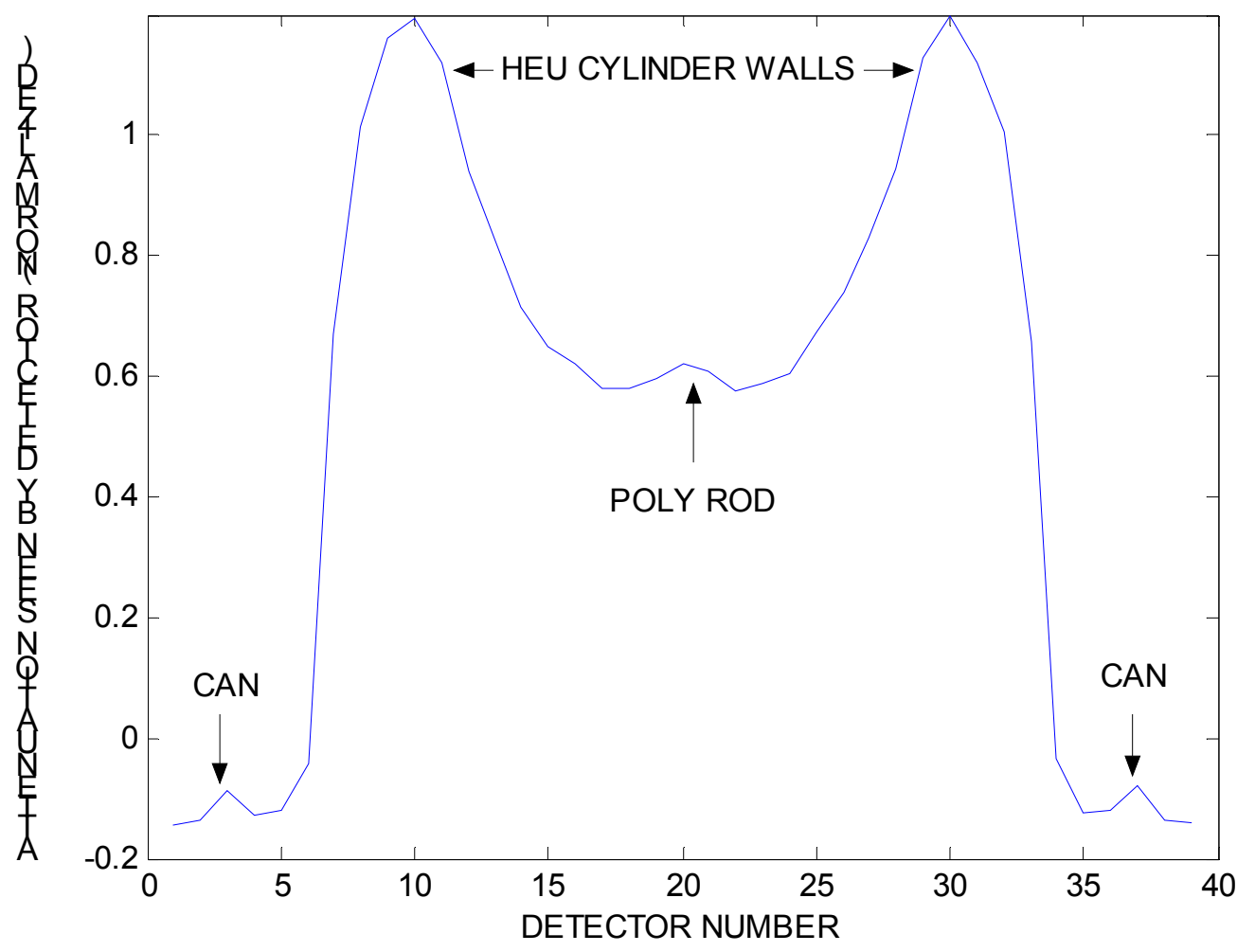

Figure 5 Detector response in terms of the radiation attenuation each detector saw with the casting in place. The portion of the NMIS signature used was predominantly neutron radiation.

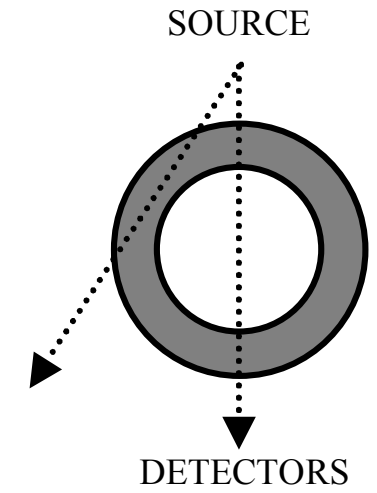

Figure 6 Transmission paths through the casting side and center.

The detectors' gamma ray response was similar except it is more highly attenuated, no poly rod effect is visible, and $\mathrm{C}$ is always less than $\mathrm{C}_{0}$. 
The tomographic reconstruction is done by the standard filtered back projection algorithm. The reconstructed image shown in Figure 7 comes from the neutron region of the NMIS signature shown in Figure 5. It shows the attenuation across a horizontal cross section of the poly rod, casting, and steel can. The gamma ray image is similar except that the poly rod is less visible. Similar results were obtained using the directlytransmitted $14 \mathrm{MeV}$ neutrons from a DT generator.

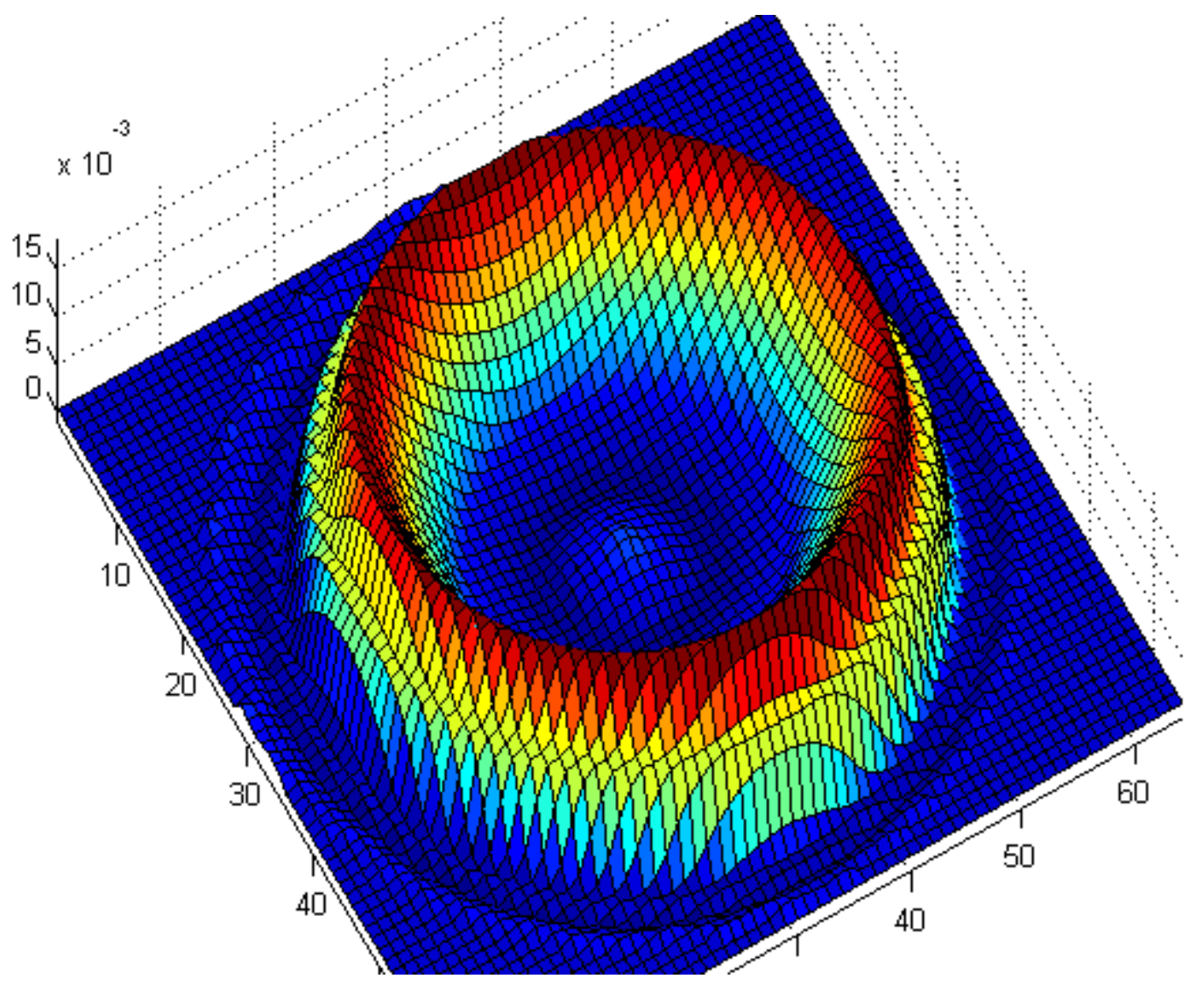

Figure 7 Reconstructed casting using the neutron portion of the NMIS signature. This reconstruction shows the attenuation measured across a horizontal cross section of the centered poly rod, the hollow HEU cylindrical casting, and the enclosing steel can.

To illustrate the use of other parts of the NMIS correlation signature, Figure 8 shows a reconstruction using only the late-arriving radiation. This radiation is a mixture of low energy source neutrons, forward-scattered source neutrons, and induced fission radiation; the latter components would cause errors in the reconstructed image. The poly rod seems to be more prominent in this reconstruction as would be expected from the lower-energy cross sections of the materials interacting with the lower energy source neutrons. However the low number of detector counts at these arrival times results in low image accuracy and a deformed poly rod shape. 


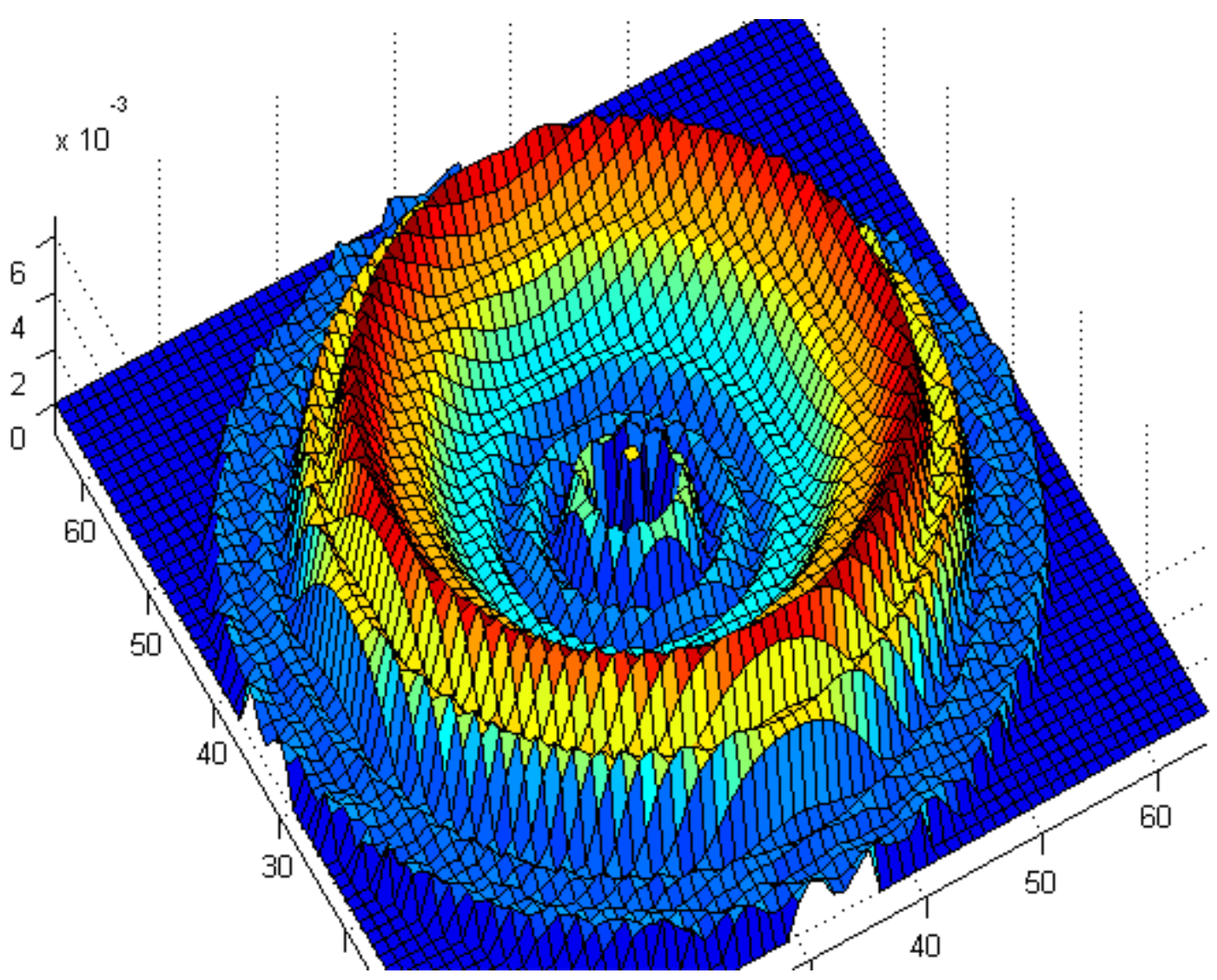

Figure 8 Reconstructed casting from the late-arriving radiation only, consisting of low energy source neutrons, scattered source neutrons, and induced fission radiation. The poly rod shows more prominently but its shape is deformed due to the low number of detector counts at these times.

Further information in the NMIS signature would be available through detector enhancements. Two enhancements under consideration are neutron - gamma discrimination and radiation energy resolution. These enhancements would allow the tomography analysis to focus more clearly on gamma rays, neutrons, and radiation with different energies.

\section{Examination of Gamma Ray and Neutron Attenuation Information}

Figure 9 shows some interesting effects by contrasting the gamma and neutron attenuation of each region in the reconstructed images. Each region is plotted at the region's neutron attenuation value ( $\mathrm{x}$ axis) and gamma attenuation value (y axis). Due to the radial symmetry of the scanned objects, the regions' properties also have radial symmetry. This causes the property curve in Figure 9 to appear to follow a trajectory that corresponds to a line from the center to the exterior of the scan.

The attenuation of any reconstructed region is the product of its materials' cross sections and density. Regions on the boundary of the HEU wall will have the HEU characteristic cross sections, but the average material density in the region will depend on how much of the region lies within the HEU and how much lies in air (void). The effect in Figure 9 is 
that the collection of regions around the HEU wall lie roughly along the line whose slope is equal to the ratio of the gamma to neutron cross sections for the ${ }^{252} \mathrm{Cf}$ energy spectrum.

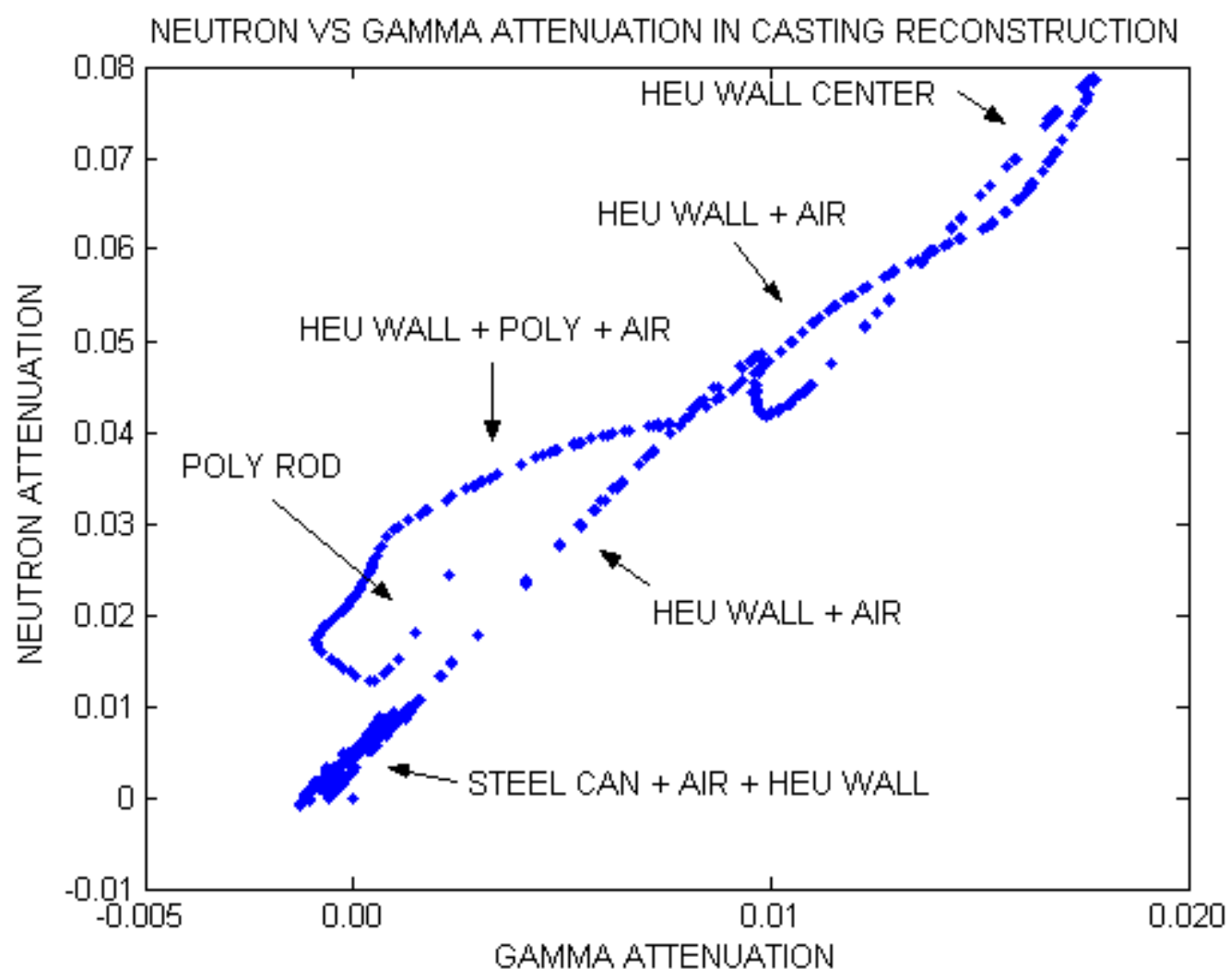

Figure 9 Gamma versus neutron attenuation properties for each region in the reconstructed image. The attenuation of each region is the product of the gamma or neutron cross section of the materials and their density averaged over the region.

The regions inside the casting are the most difficult part of this problem. The air region inside the casting is labeled in Figure 9 as "HEU + AIR + POLY". In other words, the air region's properties reflect properties of all of the surrounding materials. This is partly due to a well-know reconstruction phenomenon called beam hardening, and is illustrated in the reconstruction of bone around water in Figure $10^{4}$. The effect occurs when the source radiation with a broad energy passes through an attenuating region. More of the lower-energy radiation is removed so the emerging radiation has a harder energy spectrum; its attenuation in the remaining material it passes through is different.

\footnotetext{
${ }^{4}$ Principles of Computerized Tomographic Imaging, Avinash Kak and Malcolm Slaney, IEEE Press, New York, 1988, p 131.
} 


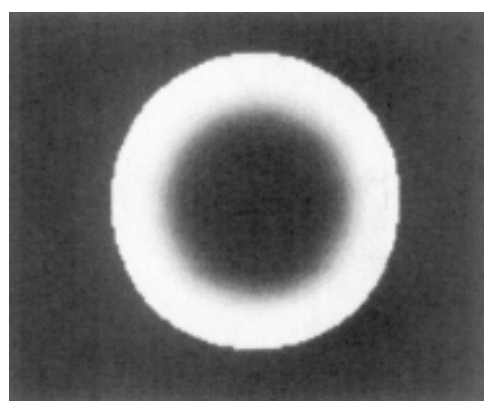

Figure 10 Reconstruction of bone around water showing the poorer definition of the interior wall due to beam hardening.

The neutron image, in particular, may also be affected by the neutrons' forward scattering from the HEU wall. Neutrons typically have small deflection angles and low energy loss when scattering from a Uranium nucleus. This would result in the neutron deflecting into a detector adjacent to the detector the neutron was originally heading towards, retaining enough energy to be counted. The effect on the image is that it would be "seen" as having come from a different source-detector ray, i.e., a different region in the casting. This decreases the resolution in the reconstructed image (blurs the regions). The sum of this and other effects is strong enough that the end detectors saw a $20 \%$ increase in counts when the casting was put in place. Gamma rays can also exhibit forward scattering.

A third effect is scattering between the detectors in the array. This configuration does not include any shielding between detectors, and it is clear from the simulations that this does occur. The effect would be that a count in the proper detector could be followed by a count in an adjacent detector and, as explained in the preceding paragraph, this will blur the regions of the image. Early simulations done with single detectors produced a sharper image.

The poly rod region is a group of points at the end of the curve with low gamma attenuation and high neutron attenuation.

There are published methods to compensate for the effects noted above that haven't been applied here because our goal is to see all of the physical effects that occur in this example. These refinements should provide a crisper image and better gamma / neutron attenuation properties determination.

MCNP-PoliMi provides other information that has not been used yet to interpret these images. It can provide radiation particle energy information, type of particle (gamma/neutron), origin of the radiation (source or induced fission), among other things.

It would be especially interesting to research ways of using the tomography analysis in combination with NMIS's DT generator signature to determine the areas of induced fission in the scanned objects. 


\section{Image Reconstruction Techniques}

The example above used the standard filtered back projection image reconstruction method. This is a simple, approximate method which can produce image artifacts, as was demonstrated by the example. The NMIS implementation should use a more flexible algebraic reconstruction technique ${ }^{5}$ (ART). ART-like techniques set up a matrix that describes how each region of the scan contributes to each detector. It might be able to simultaneously incorporate other radiation phenomenon discussed above. To a first approximation (based on just one scatter event per particle), forward scattering is a contribution from a region to adjacent detectors; induced fission is a isotropic contribution; beam hardening is a progressive hardening of the radiation energy spectrum along a source-detector ray. ART can also take advantage of prior knowledge about the item such as the fact that it is a collection of machined parts ${ }^{6}$ or the materials expected. ART is particularly useful when reconstruction must be done from few measurements, which is the main problem to solve for practical use. The main reason it is not used widely is that it is slower and memory-intensive. These problems grow quickly with resolution, but for this application the desired resolution is low $(64 \times 64$, as compared to $1024 \times 1024$ for other applications employing CCD cameras or $8 \mu \mathrm{m}$ detectors).

$\mathrm{ART}$ is an iterative technique and it has been noted that the order in which it processes the regions affects its convergence. It appears from the example above that reconstruction can start on the periphery of the scanned container, begin accurately accounting for scattering, energy spectrum shifts, etc., then move into the interior of the container while continuing to account for these effects.

\footnotetext{
${ }^{5}$ Principles of Computerized Tomographic Imaging, Avinash Kak and Malcolm Slaney, IEEE Press, New York, 1988, p 285.

${ }^{6}$ Either 2D or 3D geometries could be employed.
} 


\section{Possible NMIS Tomography Configurations}

There are several potential NMIS tomography configurations. The source could be one of three types:

1. A ${ }^{252} \mathrm{Cf}$ source providing gamma rays and neutrons as described above.

2. A DT generator operating at a few million neutrons per second or less so as to provide a source event time, plus a detector providing an arrival time, to produce the usual NMIS correlation/coincidence signature.

3. A DT generator operating at much higher rates so as to provide a high detector count rate and short measurement times. The reconstruction will use the total detector count rate instead of the full NMIS signature.

The detectors could be one of several types:

1. Standard fast plastic scintillators as currently, detecting gamma rays and neutrons indiscriminately.

2. Liquid scintillators incorporating pulse shape discrimination (PSD) to distinguish gamma rays and neutrons.

3. Liquid scintillators incorporating PSD and adding radiation energy determination to further distinguish the radiation.

Detectors might also incorporate collimators and shields.

The number of detectors affects data acquisition time and the data acquisition system. Acquisition is faster when many detectors are used simultaneously in a fan beam configuration. Otherwise detectors must be stepped through the angles the fan beam measures to produce a complete measurement. The current computer hardware interfaces to four detectors and a source; the time of each detector count is reported to the computer which produces the correlation/coincidence signature. If more detectors are used, or count rates are extremely high, it would be better to acquire the NMIS signature at the detectors. This would require a time-of-flight multi-channel analyzer circuit and a digital interface to the computer. If the source is not timed, as for a DT generator operating at very high neutron production rates, the detectors would only report total counts (or count rate).

Finally, the tomographic analysis could be high resolution in order to provide an image that an operator can easily interpret, or it might have just enough resolution to enhance the on-line analysis for attributes or template matching. The latter would have the advantage of shorter measurement times. 


\section{Summary}

The example case described in this report shows the feasibility of making a tomographic measurement with the Nuclear Materials Identification System (NMIS).

Tomography measurement and analysis is simply the current NMIS system doing additional measurements and analyzing the data more completely. Studying the tomographic application of NMIS will increase our understanding of the current NMIS system and the physical principles it is based on. Making tomographic measurements with NMIS will increase our understanding of weapons components radiation signatures.

It is too early to say exactly how it would be applied in practice. Practical considerations require a balance between a tomographic measurement and the current NMIS measurement. A tomographic measurement takes more time, while the current NMIS measurement gives most of the information required to identify a weapons component. We could imagine, for instance, a long tomographic measurement to characterize each weapon component type, then use of this characterization in day-to-day measurements made by the current NMIS method. Alternatively, a low-resolution tomography measurement could be made in day-to-day measurements and used as a signature or in a more detailed analysis based on weapon attributes. 